\title{
MONTE CARLO OF TEVATRON OPERATIONS, INCLUDING THE RECYCLER*
}

\author{
E. McCrory; Fermilab Batavia, IL, U.S.A.
}

\section{Abstract}

A Monte Carlo model depicting the operation of the Fermilab Tevatron Complex has been constructed. The purpose is to replicate the general behavior of the Complex, incorporating aspects that are known to affect its performance, in order to learn how optimally to run the Complex. The newest ring at Fermilab, the Recycler, has been added to the model in order to understand how best to incorporate it into the rest of the Complex. This model includes numerous parameterizations of the Complex, including reasonable random fluctuations and normal interruptions in operation of each accelerator due to downtime. Optimizations are performed to guide us in making using the model in order to maximize the integrated luminosity delivered to the experiments.

\section{THE MONTE CARLO MODEL}

A Monte Carlo program has been developed in the $\mathrm{C}++$ programming language to model the operational behavior of the Fermilab Tevatron Complex. The goal is to reflect accurately the present and near-future performance of the Tevatron. Then, intuition can be developed on how operations decisions affect the integrated luminosity to the experiments.

Running the Tevatron and its injectors usually requires no complicated decisions: We run when we can and do maintenance when we must. But there are some decisions that impact the amount of luminosity we deliver to the experiments. This model was created to determine an optimum for these decisions. Some of the aspects of the Complex can be modeled analytically (like the luminosity and its time dependence), but it is difficult to represent random fluctuations and downtime [1].

This model simulates the behavior of the Tevatron Complex by tracking its evolution in small time increments, 0.1 hours is typical. The detailed behavior of the model is largely phenomenological, that is, simple algorithms and formulae are used, randomizations are constructed and parameters are adjusted to reproduce the observed behavior of the Complex, including downtime. Also, observed correlations between and among measured parameters are included. For example, the model generates particle intensities and emittances (based on wellmeasured dependencies throughout the Complex), and the relationship among these values and the luminosity obtained from these values are reflected in the model.

\section{Parameterization}

The parameters in the model cover all aspects of the operation of the Complex. There are over 200 parameters in the model, and numerous relationships among them. A few of the important parameters are described here.

The Antiproton Source's Accumulator Ring has a large number of parameterizations. For example, the rate antiprotons are added to the Accumulator is:

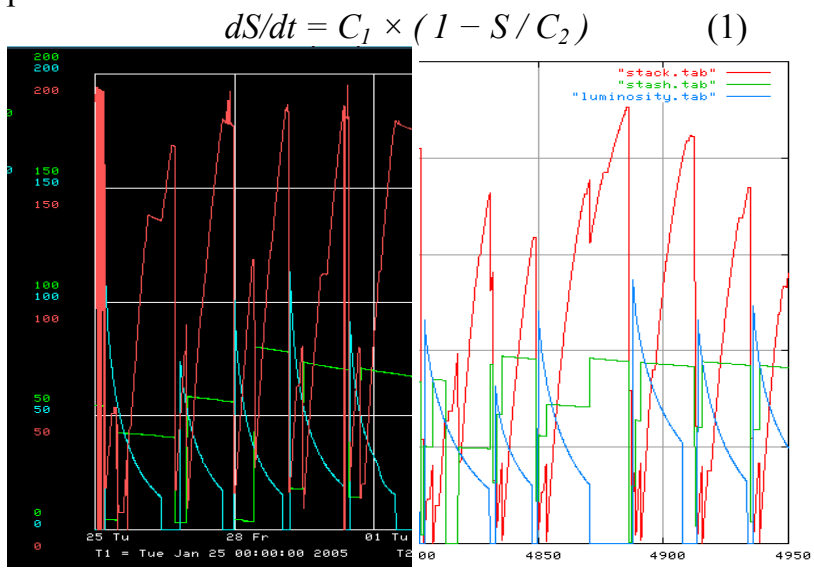

Figure 1: Real data (black) and simulated (white) of the operation of a week of the Tevatron Complex. Red: Accumulator; Green: Luminosity; Blue: Recycler.

$S$ is the stack size, $\mathrm{C}_{1}$ is called the "Zero-Stack Stacking Rate" and $\mathrm{C}_{2}$ is the stack size when the stacking rate goes to zero. These parameters are today (typically) 16 E10/hour and $300 \mathrm{E} 10$. In order to have this rate match reality, both of these parameters are randomized (the amount of randomization is itself a parameter). Additionally, the rate is allowed to diminish for one time step by up to $50 \%$ a small fraction of the time. The transverse emittance of the antiprotons extracted from the Accumulator for use in the Tevatron fall on a quadratic:

$$
\epsilon=0.00007 \times S_{\text {extr }}^{2}+0.02 \times S_{\text {extr }}
$$

$S_{\text {extr }}$ is the amount extracted for the Tevatron. The actual emittance is randomized from this by about $50 \%$.

A particularly interesting parameterization in the model is the characterization of Tevatron downtime. We observe that downtime in the Tevatron occurs randomly with respect to time, with a probability of 0.988 per hour when the Tevatron is at $980 \mathrm{GeV}$. That is, there is a $98.8 \%$ chance that the Tevatron will not failindependent of time. Moreover, this uptime is less (about $90 \%$ ) when the Tevatron is not conducting collisions.

\section{Matching to Reality}

It is not possible to model every possible real situation here - the complexities of Real Life are too varied. So we have chosen to select an extended period of good running and to match the model to it. Weeks during which there were long maintenance periods and/or substantial diversions from normal operations were ignored. The parameters of the model were adjusted to match numerous 
weekly measures of real performance, including: integrated luminosity, hours spent integrating luminosity in the Tevatron, the time spent in shot setup, downtime in each of the subsystems, total number of antiprotons accumulated, the time spent in routine studies.

The parameters have been chosen to match a good running period from the end of 2004.

The behavior of the model does not improve during a run. The intent is to model the behavior of the Complex with a frozen set of parameters. The parameters of the model are modified from run to run to show the impact of specific improvements in performance. For example, the rate antiprotons are stacked in the Accumulator has steadily improved over the history of that storage ring. But during the course of one model run, this rate is fixed at a single, user-selected function (equation 1).

\section{AN EXAMPLE}

First, we show the overall hour-to-hour performance of the Complex in Figure 1; 1a shows an actual week from 2004, and $1 \mathrm{~b}$ shows a comparable week from the model. In this figure are: The instantaneous luminosity delivered to an experiment (blue), the number of antiprotons in the Accumulator Ring (red) and the number of antiprotons in the Recycler Ring (green). We cannot replicate the exact behavior of any running period, but the general behavior is accurate.

As an example of how this model has been used, we seek an answer to the question, what is the best time to end a proton/antiproton store in the Tevatron? Before the advent of the Recycler, this was essentially the only decision available when running the Complex. An obvious choice is to end the store when it has been colliding for a specific amount of time. The model is run for this endstore algorithm using realistic values for the parameters in the Complex, as determined during the "Matching to Reality" phase. The model is run many times, each for 5000 weeks of virtual running, with different specific values for the store duration for each 5000-week run. The average integrated luminosity delivered to an experiment each week is calculated for this simulated running period, see Figure 2. The optimum target store duration by this is predicted to be 20 hours, and we should be able to integrate $16.3 \mathrm{pb}^{-1}$ each week. But longer store durations, up to about 30 hours, do not hurt much. (In this example, not all stores last the full store duration. Some stores end early due to a failure.) Experience with the real Complex has verified this behavior. Running longer stores allows us to accumulate more antiprotons, providing a buffer for unexpected situations. Running shorter stores makes the average luminosity delivered to the experiments higher. This model determines the optimum of these two opposing factors.

There are at least two other ways to end the stores that are, on average, equivalent to this. One is to end the store when the total number of antiprotons accumulated exceeds some specific value, like 150E10. Another more complicated but ultimately more robust method we call the Ratio Method. Two luminosity values are determined: The luminosity that would be obtained from the number of antiprotons accumulated, and the actual luminosity being delivered. The ratio of the expected luminosity to the actual luminosity varies with time from a small number at the beginning of a store to a large number for an old store. When this ratio exceeds a specific value (like 6), the store is terminated. Figure 3 shows the impact of the "ratio" choice on the store duration in the model.

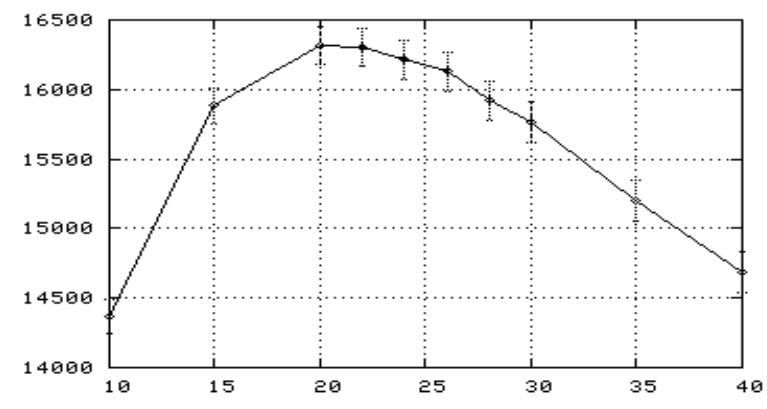

Figure 2: simulated integrated luminosity $\left[\mathrm{nb}^{-1}\right]$ vs. store duration [hours], 5000 weeks.

It is important to choose an end-store method that takes everything into account, especially the overall impact of downtime. For example, if the number of antiprotons accumulated after 20 hours is small (due to downtime, for example), it is prudent to wait longer to put in a new store. Conversely, if the store fades away particularly slowly, then the store should last longer. The ratio algorithm takes these variations into account.

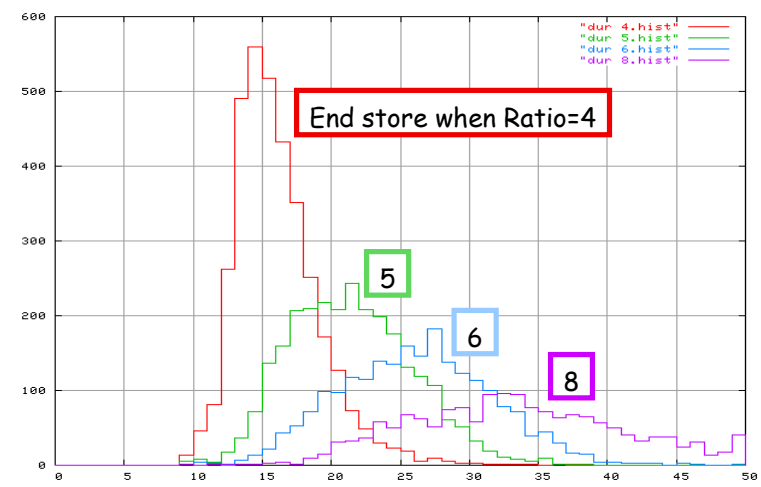

Figure 3, the store duration obtained from using the "ratio" end-store method.

This model helps predict the improvement in performance from specific changes in the behavior of the Complex, see Figure 4. For example, if we could double the reliability of the Tevatron from 0.988 to 0.994 , then the integrated luminosity we could provide to the experiments would increase by $11 \%$. If we were to double the reliability of the proton source, the reliability would only increase by $1 \%$.

\section{Some Details}

The model consists of over $80 \mathrm{C}++$ classes (for example, Tevatron, Recycler, ProtonSource, Luminosity, Random, FSM (for Finite State Machine), etc.). Each accelerator in the Tevatron Complex is represented in a 
$\mathrm{C}++$ class. Each of these classes is responsible for the time evolution of itself, and this is accomplished generically with a Finite State Machine. In general, each state of each finite state machine has a non-zero probability of transitioning to a "down" state. The meaning of "down" varies from machine to machine. For example, if the Tevatron enters the "down" state when there is a store in the machine, this often this corresponds to a quench, requiring up to several hours of recovery. But the Proton Source downtime usually is less than 10 minutes.

There are over 200 parameters that determine the precise behavior of the Complex in the model. Running on a Linux PC based on a $1.8 \mathrm{GHz}$ Celeron processor, a 5000 week run takes about 220 seconds.

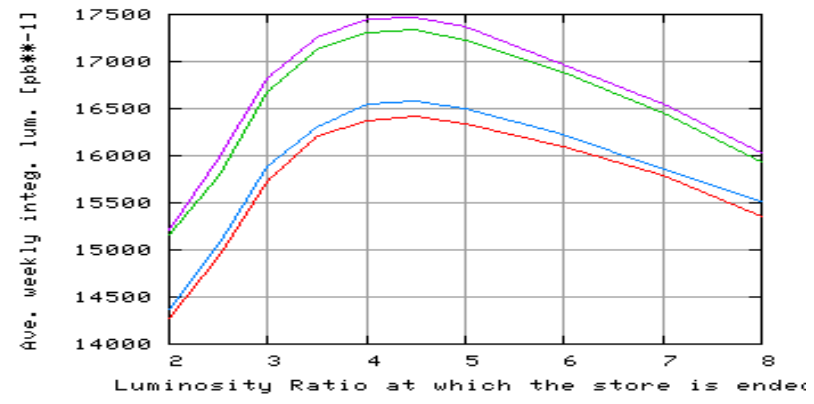

Figure 4: The impact of improvements in reliability; red: Normal; Blue: Improved proton source; Green: Improved Tevatron; Purple: Both are improved.

\section{USING THE RECYCLER RING}

The Recycler Ring has been added to the Tevatron Complex for storing antiprotons, in support of the Accumulator [2]. There are two known improvements observed from this new antiproton storage ring:

1. We can offload antiprotons from the Accumulator, allowing us to store more total antiprotons quicker.

2. The transverse emittances from the Recycler are smaller than from the Accumulator.

The performance compromises are:

1. We must stop accumulating antiprotons in order to transfer them from the Accumulator to the Recycler. It now takes 30 to 90 minutes to perform transfers.

2. Antiprotons are lost during a transfer.

3. The number of antiprotons stored is limited.

4. It has been observed that the lifetime and, occasionally, the integrated luminosity from collisions involving these brighter antiproton bunches are smaller.

These factors are incorporated into the model.

Remembering that the primary goal of the Complex is to maximize the luminosity delivered to the experiments, simulations are run to determine: (a) the best time to transfer antiprotons into the Recycler, and (b) the best time to end a store and begin a new one.

The model helps us answer these questions. Question (a) breaks down into three simpler questions: (1) How many antiprotons should we have in the Accumulator before we begin a transfer, (2) how many antiprotons do we attempt to transfer, and (3) how many transfers to the Recycler do we attempt during the course of one store?
Runs of the model show that the answer to question 2 is the intuitive one: As many as possible. Details to the answer to question 3 are beyond the scope of this paper-we will assume a maximum of 3 such transfers per store. The answer to question 1 is shown in Figure 5, where we show a third dimension, namely when do we begin an antiproton transfer from the Accumulator to the Recycler, based on the number of antiprotons in the Accumulator.

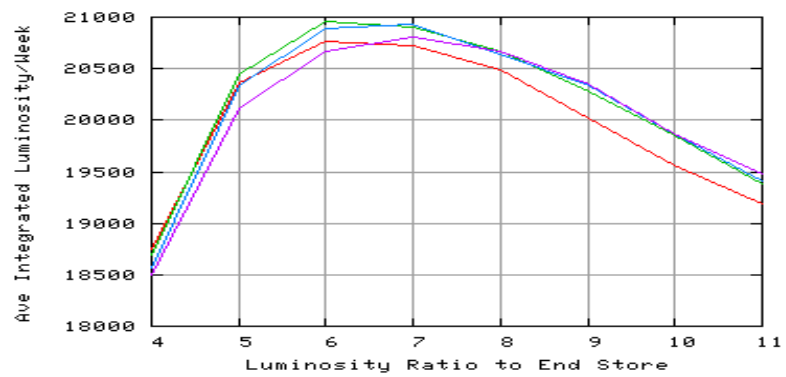

Figure 5, determining when to transfer antiprotons to Recycler; See text for description of the four lines.

There are four traces in Figure 5 which correspond to four different criteria as to when antiprotons are transferred from the Accumulator to the Recycler. They are: Red - transfer into the Recycler when the Accumulator has stored 20E10 antiprotons; Blue: 30E10; Green: 40E10; Purple: 60E10. There is a slight preference for either 30E10 or 40E10; but do not do too many transfers (20E10) and do not wait too long (60E10).

Note in Figures 4 and 5 that the ratio at which to end the store is independent of the choice of parameters: 4 to 5 without the Recycler and 6 to 7 with the Recycler.

\section{Exploring possibilities}

What if the Recycler can hold a lot more antiprotons? This question is relevant since this is a main benefit of adding electron cooling to this ring, a major upgrade project underway now [2]. Assuming that the Recycler can hold 3E12 antiprotons (the largest accumulation to date is 1.7E12), that the Accumulator can get 24E10 antiprotons per hour, and that transfers from the Accumulator to the Recycler can be made in less than 5 minutes, then it is predicted that the Tevatron Complex can approximately double the luminosity delivered to the experiments.

\section{CONCLUSIONS}

The model of Tevatron Complex operations allows us to determine how to make decisions in the Complex that optimize the luminosity delivered to the experiments. In particular, incorporating the Recycler into the Tevatron Complex increases the luminosity delivered to the experiments by about $20 \%$.

\section{REFERENCES}

[1] For details, see http://mccrory.fnal.gov/model.

[2] Sergei Nagaitsev, "Commissioning of Fermilab's Electron Cooling System for 8-GeV Antiprotons," paper \#2076 (ROPC006), this conference. 